ARTICLE

Received 30 Apr 2016 | Accepted 29 Jun 2016 | Published 12 Aug 2016

DOI: $10.1038 /$ ncomms 12403

OPEN

\title{
A robust molecular probe for Ångstrom-scale analytics in liquids
}

\author{
Peter Nirmalraj ${ }^{1}$, Damien Thompson ${ }^{2,3}$, Christos Dimitrakopoulos ${ }^{4}$, Bernd Gotsmann ${ }^{1}$, Dumitru Dumcenco ${ }^{5,6}$, \\ Andras $\mathrm{Kis}^{5,6}$ \& Heike Riel ${ }^{1}$
}

Traditionally, nanomaterial profiling using a single-molecule-terminated scanning probe is performed at the vacuum-solid interface often at a few Kelvin, but is not a notion immediately associated with liquid-solid interface at room temperature. Here, using a scanning tunnelling probe functionalized with a single $\mathrm{C}_{60}$ molecule stabilized in a high-density liquid, we resolve low-dimensional surface defects, atomic interfaces and capture Angstrom-level bond-length variations in single-layer graphene and $\mathrm{MoS}_{2}$. Atom-by-atom controllable imaging contrast is demonstrated at room temperature and the electronic structure of the $\mathrm{C}_{60}$-metal probe complex within the encompassing liquid molecules is clarified using density functional theory. Our findings demonstrates that operating a robust single-molecular probe is not restricted to ultra-high vacuum and cryogenic settings. Hence the scope of high-precision analytics can be extended towards resolving sub-molecular features of organic elements and gauging ambient compatibility of emerging layered materials with atomic-scale sensitivity under experimentally less stringent conditions.

\footnotetext{
${ }^{1}$ IBM Research-Zürich, Säumerstrasse 4, CH- 8803 Rüschlikon, Switzerland. ${ }^{2}$ Department of Physics and Energy, University of Limerick, Limerick V94 T9PX, Ireland. ${ }^{3}$ Materials and Surface Science Institute, University of Limerick, Limerick V94 T9PX, Ireland. ${ }^{4}$ Department of Chemical Engineering, University of Massachusetts, Amherst, Massachusetts 01003-3110, USA. ${ }^{5}$ Electrical Engineering Institute, École Polytechnique Fédérale de Lausanne (EPFL), CH-1015 Lausanne, Switzerland. ${ }^{6}$ Institute of Materials Science and Engineering, École Polytechnique Fédérale de Lausanne (EPFL), CH-1015 Lausanne, Switzerland. Correspondence and requests for materials should be addressed to P.N. (email: pni@zurich.ibm.com).
} 
S ensing objects below the resolution limit of the eye began with the advent of water-based microscopes in 2000 BCE. Following a series of ground-breaking advances, instrument development eventually led to the modern electron and scanning probe microscopes which have not only advanced resolution capability but have enabled new research disciplines from quantum cryptology to single-molecular nanotechnology. Recently, high-resolution transmission electron microscopy ${ }^{1}$, scanning tunnelling microscopy (STM $)^{2-4}$ and scanning tunnelling spectroscopy ${ }^{5}$ have made possible the analysis of a new class of ultra-thin materials. The atomic-scale structure, extrinsic doping, bonding states and chemical composition can be precisely measured for single-atom-thick electronic materials from the two-dimensional (2D) form of carbon, graphene ${ }^{6}$ to more recent transition metal dichalcogenides (TMDs) ${ }^{7}$ with potential for triggering a new wave of 2D nanodevice technologies.

However, preserving the surface integrity of single-atom-thick materials outside of ultra-high vacuum (UHV) settings, and extracting information with highest possible resolution, where each atom is directly exposed to contaminants is not trivial. Yet the payoff is immense. To take one illustrative example, it would allow rapid fingerprinting of a vast body of theoretically predicted $2 \mathrm{D}$ layered materials ${ }^{8}$ and simultaneously verify the ambient compatibility of such novel materials ${ }^{9}$ and $2 \mathrm{D}$ patterned structures ${ }^{10}$. A known alternative to vacuum to protect air-sensitive surfaces, is the liquid-solid interface in which a STM can be operated. This field of research has an almost 30-year history, from early reports on achieving atomic resolution on solid surfaces immersed in water ${ }^{11}$, liquid nitrogen ${ }^{12}$ and acidic solution $^{13}$ environments, observation of molecular dynamics ${ }^{14}$, decoding molecular layer-underlying surface epitaxial relationship $^{15}$, capturing oxidation catalysis reactions ${ }^{16}$ and in investigating through high-resolution STM images the supramolecular chemistry of molecules ${ }^{17}$ and pattern formation during molecular self-assembly ${ }^{18}$ at the liquid-solid electrical interface with a non-functionalized metal probe. More recently it has been demonstrated that by terminating the apex of a scanning probe (including STM and AFM) with a single molecule, it is possible to further the limits of spatial resolution and enhance chemical contrast of the low-dimensional materials under study ${ }^{19-26}$. However, these single-molecule-terminated scanning probes have been mainly demonstrated to operate at UHV in a temperature scale ranging from 4 to $100 \mathrm{~K}$ (refs $20-22,25,27,28$ ). The protocols for engineering such molecular probes, in particular bonding effects between the molecule and the metal-apex, have been documented in detail $22,24,27-30$. Conversely, the benefits of the molecular probe have not been fully exploited under standard laboratory conditions, mostly because of instabilities in moleculemetal coupling at room temperature. In the present work, we demonstrate that by engineering and operating a single-moleculeterminated Au STM probe in high-density liquids, it is possible to control random fluctuations of the molecule at the metal apex, maintain a clean interface protected from ambient contaminants thereby resulting in a robust single-molecular probe with prolonged lifetime.

\section{Results}

Electron tunnelling in a high-density liquid environment. The central concept of our experiment is depicted in Fig. 1, a metallic (Au) STM probe functionalized with a $\mathrm{C}_{60}$ molecule is brought close to the surface (epitaxial graphene on $\mathrm{SiC}^{31}$ ) held in a Teflonbased liquid cell filled with silicone oil $\left.\left(\left[-\mathrm{Si}\left(\mathrm{CH}_{3}\right)_{2} \mathrm{O}\right)-\right]_{n}\right)$. $\mathrm{C}_{60}$ geometry permits easier lateral manipulation and transfer to the tip from the surface at ambient conditions than, for example, $\mathrm{CO}$, and the protocol for $\mathrm{C}_{60}$ transfer is well

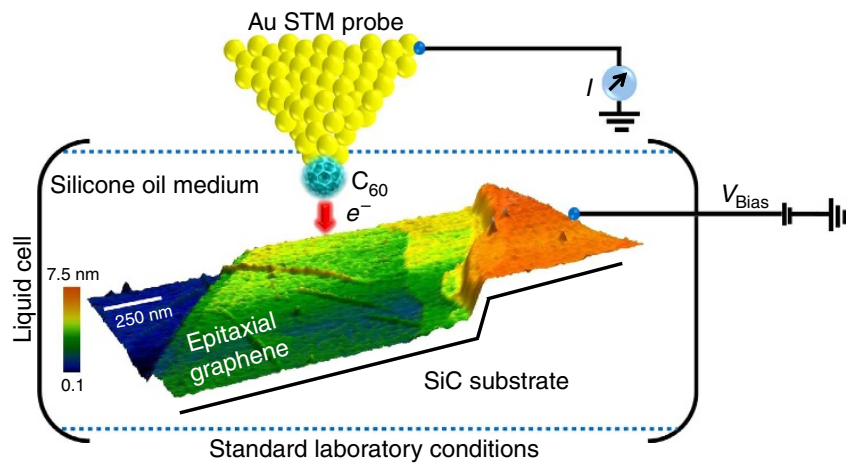

Figure 1 | Description of the liquid-based STM experimental design. Schematic of the liquid-cell setup with the single-molecule-terminated $\mathrm{Au}$ tip (connected to an external current pre-amplifier circuit) positioned over an epitaxial graphene sample (on which the bias voltage is applied). The entire liquid cell holds an electrochemically inert and high-density silicone oil at room-temperature conditions. The liquid cell is based on Teflon.

established ${ }^{19,22,24,32}$. Single $\mathrm{C}_{60}$-terminated STM tips are routinely used to probe organic materials and $\mathrm{C}_{60}$ clusters anchored on metal STM tips have also been demonstrated previously, to investigate low-dimensional surface defects ${ }^{33}$. Such molecular cluster-based tips are relatively robust (loss of a single molecule at the apex can be compensated by its nearest neighbour) but interpretation of the observed chemical contrast improvements is not trivial. The mechanism of electron tunnelling from the $\mathrm{C}_{60}$-terminated $\mathrm{Au}$ probe through a non-polar liquid medium into the conductive graphene layer may be modelled as tunnelling through a vacuum gap where the tunnelling current $\left(I_{\text {tunnel }}\right)$ is exponentially dependent on the distance $(Z)$ between the probe and the conductive surface using an effective energy barrier height $\left(\phi_{\text {eff }}\right)$ as shown below,

$$
I_{\text {tunnel }} \sim \frac{V_{\text {bias }}}{Z} \exp \left(- \text { const } \sqrt{\Phi_{\text {eff }}} Z\right)
$$

However, the effective energy barrier height ( $\left.\phi_{\text {eff }}\right)$ has been shown to fluctuate for STM measurements at the conductive liquid-solid electrical interface ${ }^{34}$. In addition to fluctuations related to $\phi_{\text {eff }}$, the tunnelling probability (both elastic and inelastic) during STM measurements in polar liquids can be influenced by the barrier dielectric response. To exclude these effects, it is necessary to work in a liquid that is electrochemically inert, has a low affinity towards the sample and a low vapour pressure. The dense molecular structure of silicone oil prevents moisture build-up on the solid surface, protects air-sensitive samples ${ }^{12,35}$ and minimizes tip and surface contamination ${ }^{36}$. Note that during the tunnelling process (irrespective of bias polarity), the liquid can be squeezed out of the gap and this holds for both polar ${ }^{37}$ and non-polar tunnel barriers ${ }^{2,35,38}$.

Engineering and testing the robustness of the molecular probe. Single $\mathrm{C}_{60}$-terminated metal STM tips have been fabricated in the past through lateral manipulation and transfer of a surface bound $\mathrm{C}_{60}$ molecule to a metal apex under cryogenic conditions ${ }^{22}$ and later applied to resolve intramolecular details of molecules ${ }^{19}$ and applied in atom-scale engineering of single-molecule contacts ${ }^{24}$. We attempt a challenging lateral manipulation technique to engineer a $\mathrm{C}_{60}$ functionalized $\mathrm{Au}$ probe in a liquid environment at room temperature. For this we first locate an isolated $\mathrm{C}_{60}$ molecule on $\mathrm{Au}(111)$ surface in silicone oil liquid cell and the specific molecule was later transferred to the metal tip by controlled lateral manipulation as shown from the schematic steps (step 1-step 4) in Fig. 2a. The tunnel current set point is 
reduced by nearly $30 \%$ during transfer attempt in comparison to the set point applied during normal imaging experiments. To verify whether the lateral manipulation approach was successful, the same region is scanned again. The target molecule indicated by the black arrow in the STM image (Fig. 2b) and after the lateral manipulation (Fig. 2c) experiment in the presence of the liquid medium, serves as a first proof of the transfer of a $\mathrm{C}_{60}$ molecule from the surface to the Au tip. However, we did not observe any immediate apparent change in the structure of the near-lying $\mathrm{C}_{60}$ molecule when imaged with a $\mathrm{C}_{60}-\mathrm{Au}$ probe as shown in Fig. 2c, which was recorded at a high speed (scan rate of $30.5 \mathrm{~Hz}$, tip velocity of $15.3 \mu \mathrm{m} \mathrm{s}^{-1}$ and $256 \times 256$ lines per scan area). This is in contrast to previous low-temperature STM studies of $\mathrm{C}_{60}$-terminated tungsten tips ${ }^{22}$, where directly after termination of the surface bound molecule to the metal apex resulted in visualization of the molecular orbitals of the $\mathrm{C}_{60}$ when imaged over $\mathrm{Au}$ adatoms, which served as sharp reverse imaging sites. In our study, upon reducing the scan speed (scan rate of $1.51 \mathrm{~Hz}$, tip velocity of $0.7 \mu \mathrm{m} \mathrm{s}^{-1}$ and $512 \times 512$ lines per scan area), we obtained an improved visualization of the structural change incurred on the surface bound $\mathrm{C}_{60}$ molecule (Fig. 2e), when compared with the featureless structural shape imaged previously for the same molecule with a non-functionalized Au tip (Fig. 2d). After passing this control test, the molecular probe $\left(\mathrm{C}_{60}-\mathrm{Au}\right.$ STM tip) was then directly transferred to a liquid-cell holder with the epitaxial graphene sample encompassed in silicone oil. Here again we conduct an additional control test to confirm the presence of the molecular probe, which is a reverse imaging experiment at local defect sites on graphene. The observed structural changes were comparable to early $\mathrm{C}_{60}$-terminated STM-based reverse imaging studies on defects on a graphite surface ${ }^{39}$, which further validates the presence of the $\mathrm{C}_{60}$ molecule at the Au tip-apex. (see 'Methods' section and Supplementary Fig. 1, Fig. 2a,b). Note: In total, we have conducted 135 attempts to fabricate the $\mathrm{C}_{60}$ molecular STM probe through lateral manipulation, of which $\sim 80$ were successful. This nearly $65 \%$ success rate includes tests where we were in the process of finding the optimal tip-sample height distances and bias energy ranges. Generally the failed attempts were due to incidents where the bias energy $(\geq 1.8 \mathrm{~V})$ and/or the current set point ( $\geq 250 \mathrm{pA}$ ) was too high, during the manipulation attempt, suggesting that the molecule was displaced rather than transferred. Note: The optimal bias energy range and tunnel current set point values apply only during manipulation
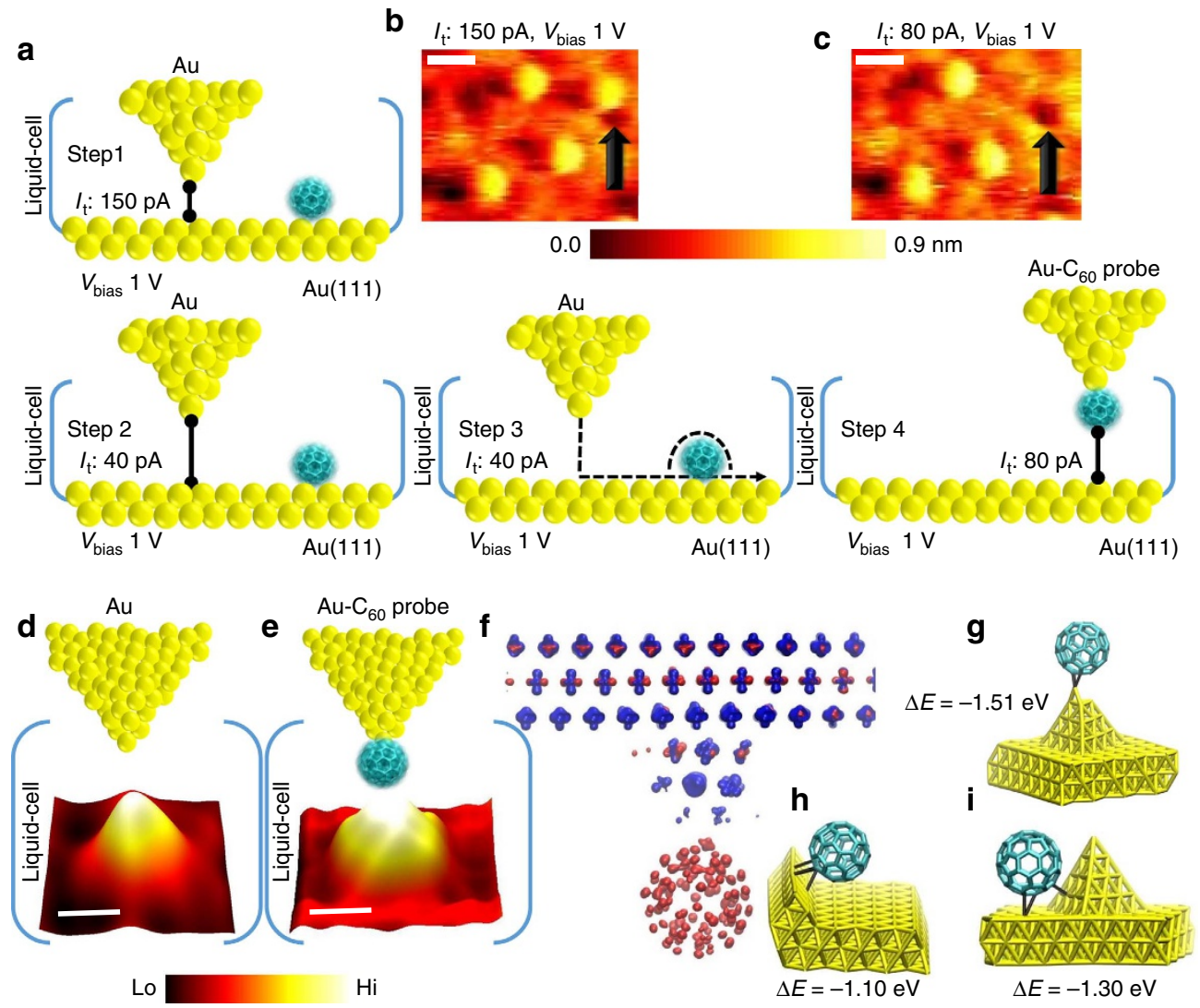

Figure 2 | Fullerene terminated STM probe formation, analysis and electronic structure. (a) Schematics detailing the steps involved in formation of a molecular probe by controlled transfer of a surface confined molecule to the apex of the Au probe. (b) Constant-current STM image of isolated $\mathrm{C}_{60}$ molecules adsorbed on $\mathrm{Au}(111)$. The molecule marked by the black arrow is selected for the transfer. (c) Imaging the same area after the lateral manipulation transfer experiment. The scale bars in $\mathbf{b}$ and $\mathbf{c}$ are $1.1 \mathrm{~nm}$. (d,e) Control experiments in which the individual molecule on the surface is imaged using a $\mathrm{Au}$ probe (d) and $\mathrm{C}_{60}$-terminated $\mathrm{Au}$ probe $(\mathbf{e})$, showing different structural lineshape with the $\mathrm{C}_{60}$ probe revealing submolecular structure. (Tunnelling parameters for data shown in $\mathbf{d}$ and $\mathbf{e}$ are $I_{\text {tunnel }}\left(I_{t}\right)=50 \mathrm{pA}, V_{\text {bias }}\left(V_{\mathrm{b}}\right)=0.5 \mathrm{~V}$. The scale bars in $\mathbf{d}$ and $\mathbf{e}$ are $0.8 \mathrm{~nm}$. (f) Computed electron density surface for the molecular probe in silicone oil showing electron density maps of the occupied states at the top of the valence band (coloured blue, calculated over the energy range of $-1 \mathrm{~V}$ up to the Fermi energy, set to $\mathrm{OV}$ ) and unoccupied states at the bottom of the conduction band (coloured red, calculated in the energy range of $0 \mathrm{~V}$ to $+1 \mathrm{~V}$ ). The isovalue used is 0.05 and the atoms are removed for clarity. (g-i) Electronic structure calculations of representative bonding sites of the $\mathrm{C}_{60}$ molecules on nanostructured gold. Au-Au and $\mathrm{Au}-\mathrm{C}$ distances below 3.2 and $3.0 \AA$ are drawn as sticks and $\mathrm{C}-\mathrm{C}$ distances below $2.0 \AA$ are drawn as sticks. $\Delta \mathrm{E}$ is the computed $\mathrm{C}_{60}-\mathrm{Au}$ bond energy. 
experiments, the molecular probe after fabrication can be operated in a bias energy range of $-2 \mathrm{~V}$ to $+2 \mathrm{~V}$ and under a tunnel current set point range of a few pA to $\sim 1.5 \mathrm{nA}$. However, the best imaging on 2D materials was obtained at low bias and low tunnel current set points and this also extended the lifetime of the molecular probes.

Similar transfer experiments in the absence of the silicone oil liquid medium had a much lower success rate of $10 \%$ and even the few molecular probes that we were able to synthesize did not last for more than a few scans under dry conditions, indicating the importance of the encompassing liquid medium in reducing the fluctuations of the molecule at the Au tip-apex. To further quantify the bonding mechanism of the $\mathrm{C}_{60}$ molecule to the $\mathrm{Au}$ tip, we rely on quantum mechanical models (representative structures shown in Fig. 2g-i, also see Supplementary Methods 1 for details of the density functional theory (DFT) calculations) which indicate that the $\mathrm{C}_{60}$ molecule exhibits a significant preference of $(0.35 \pm 0.03) \mathrm{eV}$ per molecule for bonding to low-coordination defect sites rather than terrace sites on $\mathrm{Au}$ and reveal that the strongest covalent character for $\mathrm{C}_{60}$ attachment occurs at the apex site (by contrast, terrace sites form predominantly van der Waals attachments to $\mathrm{C}_{60}$ ).

Atom-scale metrology of graphene systems. Figure 3a is a largearea STM image acquired on a graphitic region. The interface (marked by the black dotted line) separates the monolayer region (marked by the blue arrow) and bilayer region (marked by the green arrow). The area marked exactly at the interface (indicated by the white rectangle in Fig. 3a) is projected in a three-dimensional format as shown in Fig. $3 \mathrm{~b}$. The content of the imaged structure (Fig. 3a,b) is in agreement with previous UHV-STM experiments performed on similar graphene systems at $4 \mathrm{~K}$ (ref. 40) and high-resolution AFM studies at room temperature ${ }^{41}$. Zooming into the separate regions indicates clearly the bilayer structure (Fig. 3c) and monolayer graphene with a honeycomblike architecture (Fig. 3d). A three-dimensional representation of a similar monoatomic graphene lattice (Fig. 3e) highlights the atomic hillock-and-valley structures, signifying that these $2 \mathrm{D}$ materials are not perfectly planar and can have differences in the local corrugations. Imaging on a separate region on the monoatomic graphene lattice, we also observed point defects exhibiting a two-fold symmetry (Fig. 3f) as previously observed on monolayer graphene imaged in a UHV-based STM using nonfunctionalized metal tips ${ }^{42}$. The occurrence of corrugations and point defects, appearing as protrusions on the atomic lattice, is dependent not only on the synthesis mechanism but relates strongly with the registry of these materials to the underlying surface and its roughness ${ }^{43}$. The coupling of the $\mathrm{C}_{60}$ molecule to the metal-apex within the silicone oil medium is key to the stability of these molecular probes under ambient conditions. Control experiments where the molecular probes were used to image graphene (with similar bias and tunnel current set points) but under dry-conditions lasted for only a few scans (five to eight frame scans at a rate of $\sim 15 \mathrm{~s}$ per frame, after which the molecule was lost, confirmed through experiments conducted on isolated $\mathrm{C}_{60}$ molecules and reverse imaging ${ }^{33}$ over lattice defects on graphene, see section 1 Supplementary Information). This is in contrast to the longer lifetime of the molecular probes in silicone oil ( $\sim 60$ frame scans of comparable scan areas at a rate of $\sim 15 \mathrm{~s}$ per frame, and in a few cases longer than 60 frame scans when applied for imaging over graphene regions free of defects and protrusions).

Quantifying bond lengths and molecular fluctuations. Figure 4a is a STM close-up view of the hexagonal honeycomb architecture. The disparity in the bond lengths connecting the monolayer of carbon atoms can be directly visualized and quantified, thus highlighting the capability of the molecular probe to operate in a

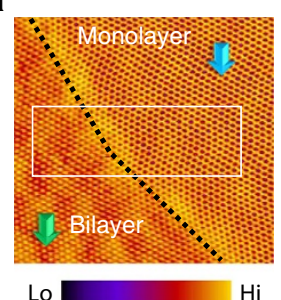

C

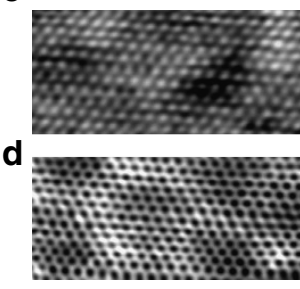

Lo $\mathrm{Hi}$ b

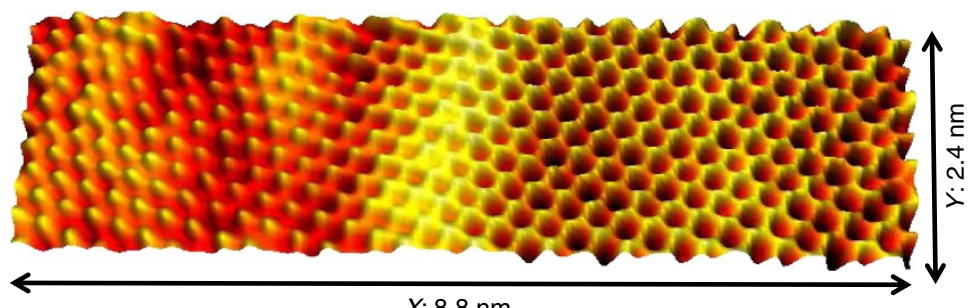

$X: 8.8 \mathrm{~nm}$

e

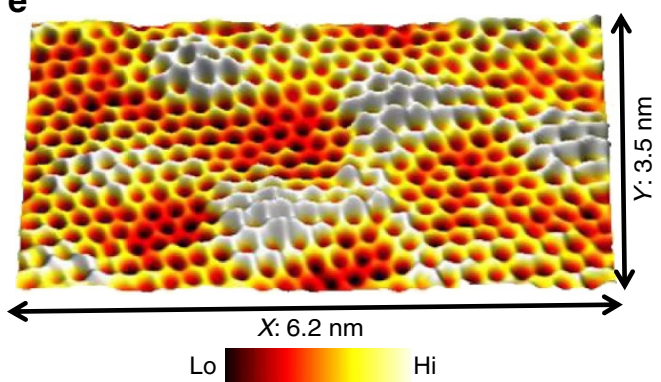

f

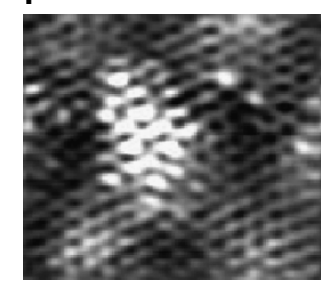

Lo $\mathrm{Hi}$

Figure 3 | Atomistic metrology of epitaxial graphene in liquid environment. (a) Wide-angle constant-current STM image revealing monolayer and bilayer graphene regions. The black line indicates the interface between the regions (Tunnelling parameters: $I_{\mathrm{t}}=85 \mathrm{pA}, V_{\mathrm{b}}=1.1 \mathrm{~V}$, scan size: $9.4 \mathrm{~nm} \times 8.7 \mathrm{~nm}$ ). (b) High-resolution constant current STM image showing the bilayer and monolayer regions within a single frame. (Tunnelling parameters: $I_{\mathrm{t}}=65 \mathrm{pA}$, $\left.V_{b}\right)=1.3 \mathrm{~V}$ ). (c,d) Constant-current STM image showing atomically clean lattice of bilayer and monolayer graphene, respectively. (Tunnelling parameters for $\mathbf{c}: I_{\mathrm{t}}=100 \mathrm{pA}, V_{\mathrm{b}}=0.9 \mathrm{~V}$, scan size: $5.2 \mathrm{~nm} \times 2.5 \mathrm{~nm}$; and for d: $I_{\mathrm{t}}=100 \mathrm{pA}, V_{\mathrm{b}}=1.1 \mathrm{~V}$, scan size: $5.8 \mathrm{~nm} \times 2.7 \mathrm{~nm}$ ). (e) STM image highlighting local protrusions and depressions in single-layer graphene $\left(I_{t}=100 \mathrm{pA}, V_{b}=0.9 \mathrm{~V}\right.$, scan size: $\left.6.2 \times 3.5 \mathrm{~nm}\right)$. (f) High-resolution constant-current $\mathrm{STM}$ image of point defect on monolayer graphene lattice $\left(I_{t}=25 \mathrm{pA}, V_{\mathrm{b}}=0.3 \mathrm{~V}\right.$, scan size: $\left.2.7 \mathrm{~nm} \times 2.5 \mathrm{~nm}\right)$. Scan speed parameters for $\mathbf{a}$, $\mathbf{c}$, $\mathbf{d}$ and $\mathbf{f}$ were maintained at $8 \mathrm{~Hz}$ at $512 \times 512$ lines per scan and for $\mathbf{b}$ and e were recorded with a scan speed of $2 \mathrm{~Hz}$ at $512 \times 512$ lines per scan. 
a

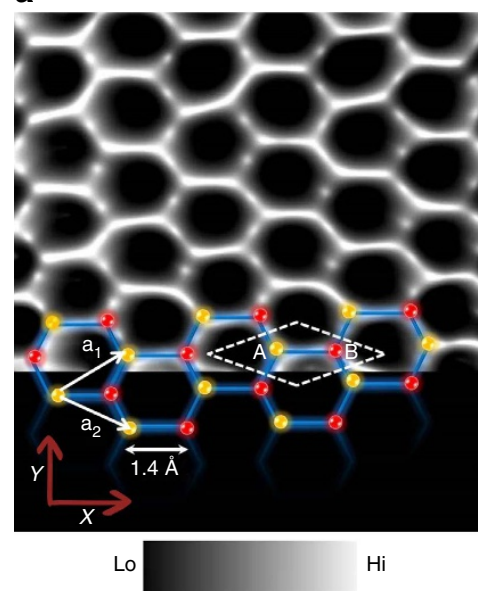

b
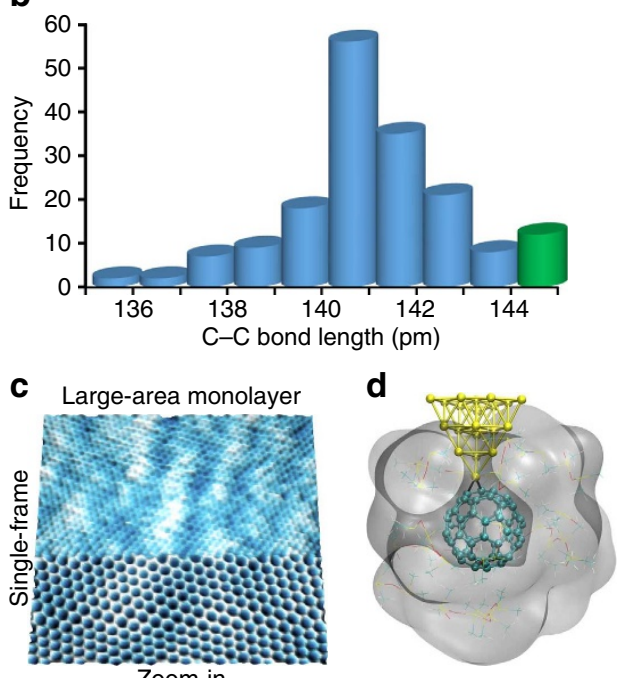

Zoom-in

Figure 4 | Liquid-stabilized molecular probe for bond length quantification. (a) High magnification constant-current STM image within a graphene monolayer region acquired using a single $\mathrm{C}_{60}$ molecular probe showing the local arrangement of the atomic lattice of graphene $\left(I_{\mathrm{t}}=200 \mathrm{pA}, V_{\mathrm{b}}=0.5 \mathrm{~V}\right.$, scan size: $1.7 \mathrm{~nm} \times 1.2 \mathrm{~nm}$ ). The yellow and red spheres point to the two equivalent carbon atoms present within a primitive unit, indicated by the white dashed rhombus and lattice vectors $\mathrm{a}_{1}$ and $\mathrm{a}_{2}$, within a monoatomic $s p^{2}$ bonded network of graphene. (b) Statistical distribution of $\mathrm{C}-\mathrm{C}$ bond lengths measured using a $\mathrm{C}_{60}$-molecular probe on a monolayer of graphene spanning an area of $\sim 200 \mathrm{~nm}^{2}$. The statistical distribution shown in $\mathbf{b}$ is based on systematic analysis over a large-area monolayer $(90 \mathrm{~nm} n \mathrm{~m} \times 125 \mathrm{~nm})$ followed by zoom-in $(11.3 \mathrm{~nm} \times 10.3 \mathrm{~nm}$, on specific locations to extract the $\mathrm{C}-\mathrm{C}$ bond distance values $(\mathbf{c})$. The scanning parameter remain constant, $\left(I_{t}\right)=155 \mathrm{pA},\left(V_{b}\right)=0.6 \mathrm{~V}$. The upper $\mathrm{C}-\mathrm{C}$ bond length values displayed in the histogram coded in green were measured using the molecular probe on atomic hexagons near the vicinity of point defects on monolayer graphene. Scan speed parameter for a and $\mathbf{c}$ was $2 \mathrm{~Hz}$ and $12 \mathrm{~Hz}$ at $512 \times 512$ lines per scan, respectively. (d) Snapshot of the final simulated tip structure obtained after $50 \mathrm{~ns}$ of equilibrated room temperature molecular dynamics of the $\mathrm{C}_{60}$-functionalized gold tip in silicone oil. Silicone oil molecules within $5 \AA$ of $\mathrm{C}_{60}$ are shown as lines with oxygen atoms coloured red, hydrogen white, carbon cyan and silicon yellow, and space-filling surface is overlaid (radius scale 1.2, density isovalue 1.0 and grid spacing 0.5 -generated using visual molecular dynamics (VMD) software). More distant silicone oil molecules are omitted for clarity.

liquids. We calculated the mean bond length connecting two adjacent carbon atoms within an atomic hexagon to be $1.41 \AA$, consistent with previous high resolution STM reports performed under cryogenic conditions ${ }^{44}$. The measured bond length is double the atomic radius of carbon $(0.7 \AA)$. Factors such as substrate curvature, topological defects and surface corrugations can induce strain, leading to variations in the experimentally measured bond lengths. The upper limit of the bond length values we report (coded in green in the histogram, Fig. 4b) was mainly measured near point defects on the graphene lattice, particularly in the vicinity of a topological defect region (similar to the constant current STM data on a point defect in Fig. 3f). This is consistent with previous theoretical calculations predicting an asymmetric bond length distribution in the graphene lattice at room temperature ${ }^{45}$ and with previous high-resolution transmission electron microscopy analysis of $\mathrm{C}-\mathrm{C}$ bond length fluctuations in single-layer graphene ${ }^{46}$.

To better understand the role of silicone oil in the formation of a stable molecular probe, we performed classical molecular dynamics simulations to examine the motion of $\mathrm{C}_{60}$ on the metal-apex in the presence and absence of the liquid. Single $C_{60}$ dynamics on the tip-apex is expressed as root-mean-square fluctuations in the $\mathrm{C}_{60}$ centre of mass, averaged over 4,000 structures sampled (every $10 \mathrm{ps)}$ ) during the final $40 \mathrm{~ns}$ of $50 \mathrm{~ns}$ of equilibrated room-temperature dynamics. The molecular dynamics model cell was based on DFT calculations of $\mathrm{C}_{60}$ bonding to an $\mathrm{Au}$ apex site via two $\mathrm{Au}-\mathrm{C}$ bonds of length $2.2 \AA$ (Fig. 4d). The $\mathrm{C}-\mathrm{Au}$ bond force constants of $55 \mathrm{~N} \mathrm{~m}^{-1}$ are estimated from DFT by shifting the $\mathrm{C}_{60}$ molecule $0.25 \AA$ closer and nearer to $\mathrm{Au}$ in steps of $0.05 \AA$, consistent with previous reports ${ }^{30}$. Figure $4 \mathrm{~d}$ is a snapshot of the final computed structure of the $\mathrm{C}_{60}$-functionalized $\mathrm{Au}$ probe in silicone oil. The computed $\mathrm{C}_{60}$ dynamics on the Au probe revealed significant reduction of $\mathrm{C}_{60}$ motion on the Au tip-apex in silicone oil. There is a threefold damping when silicone oil covers the molecular probe (see Supplementary Methods 2 for more details of the molecular dynamics simulations).

Tuning atom-by-atom imaging contrast. Having established a route to resolve with high spatial clarity the covalent bonding sequence at a fixed bias energy, we then posed the question, is it possible to control the imaging contrast at a single-atom level? Figure $5 \mathrm{a}-\mathrm{d}$ is a series of constant current STM images acquired over a single atomic hexagon spanning an area of $4.2 \AA^{2}$ represented in a three-dimensional format, imaged at a fixed tunnel current set point $(15 \mathrm{pA})$ and increasing the bias energy in steps. We observe a marked improvement in the ability of the molecular probe to identify the exact location of the atoms (Fig. 5a-d) within a six-membered carbon hexagonal ring. The location of the atomic sites can be controllably revealed and masked by increasing and decreasing the bias energy at fixed tunnel current. This level of control and ability to tune the imaging contrast, permits direct visualization of the electronic variations in embedded atoms, in this case the inter-atomic bonds within a single hexagon. We believe the high spatial information content upon terminating the $\mathrm{Au}$ tip with a $\mathrm{C}_{60}$ molecule and bias-dependent control of contrast can be attributed to the corresponding alterations in the tip states, magnitude of the bias energy and the resulting changes in the spatial orbital overlap between the molecular probe and monoatomic graphene. The local electrostatic interactions ${ }^{47}$ between the molecule and 


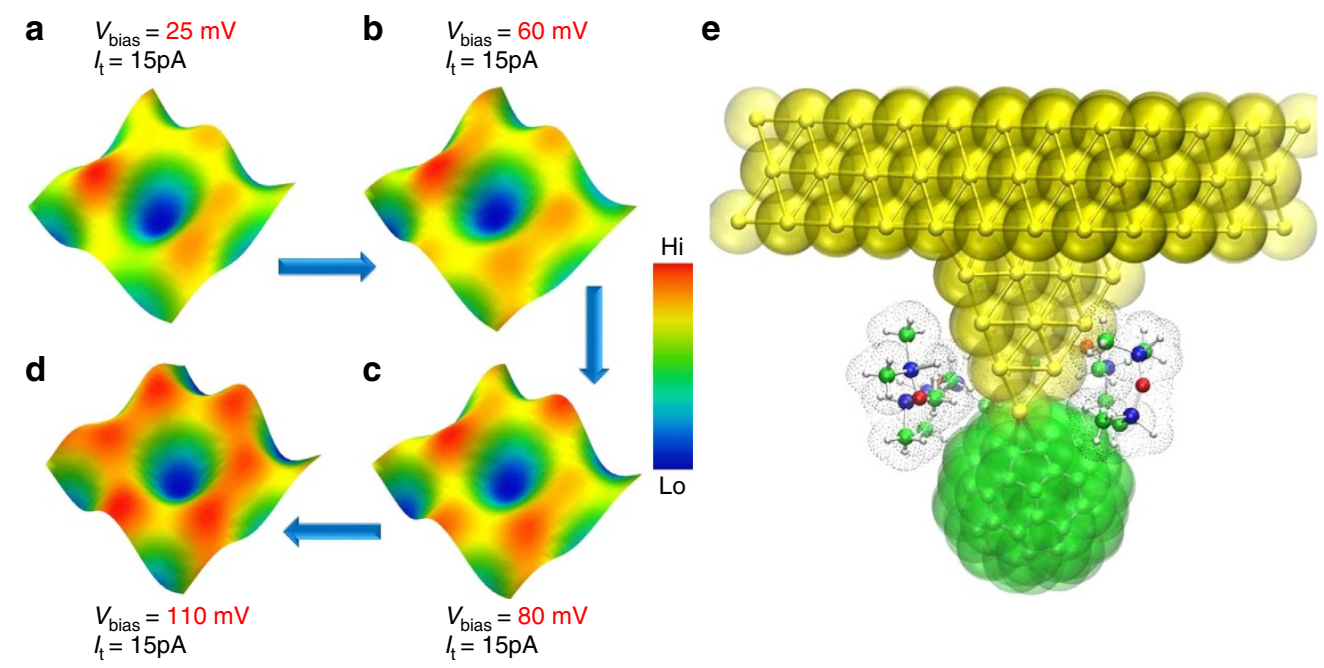

Figure 5 | Tuning atom-by-atom imaging contrast with a single-molecular probe. (a-d) Series of three-dimensionally represented constant-current STM images over an atomic hexagon recorded under incremental bias steps at a fixed tunnel current set point. The positions of all six carbon atoms within a hexagonal ring are directly resolved as shown in $\mathbf{d}$. Scan speed parameters for a-d are maintained at $2 \mathrm{~Hz}$ at $512 \times 512$ lines per scan. (e) The calculated electronic structure of the molecular probe complex with silicone oil molecules coordinating the $\mathrm{C}_{60}$-gold interface. Atoms are shown in ball and stick representation with space-filling spheres overlaid (dotted surface used for solvent). Au atoms are coloured gold, $\mathrm{C}$ atoms are green, $\mathrm{Si}$ atoms are blue, $\mathrm{O}$ atoms are red and $\mathrm{H}$ atoms are coloured white. The computed solvent structure is shown in an alternative orientation in Supplementary Fig. 7, together with more details of the local and extended solvent structure around the probe.

the graphene surface is also a factor that contributes to the enhancement in imaging contrast. To gain a deeper quantitative understanding of the impact of tunnelling parameters on the STM images in a liquid medium, it is requisite to establish a first principle-guided study of electron tunnelling between a molecular probe and graphene lattice in the presence of a silicone oil mesh similar to previous studies on electronic tunnelling through polar liquids ${ }^{48}$.

Although we have been able to previously image the atomic lattice of single-layer graphene in liquids using non functionalized metallic tips ${ }^{36}$, with resolution comparable to the data shown in Fig. 3d, it has not been possible to achieve the resolution and imaging control shown in Fig. $4 \mathrm{a}$ and Fig. 5a-d using a bare metallic Au tip in liquids at room temperature. Such high resolution imaging where the exact atomic positions can be identified allows better quantification of bond atomic distances in $2 \mathrm{D}$ crystals that is closer to the expected theoretical estimates. To check for any electronic interference from the silicone oil medium in the observed variations in imaging contrast, we performed dispersion-corrected electronic structure calculations on the $\mathrm{C}_{60^{-}}$ terminated Au probe in the presence of silicone oil molecules. The $\mathrm{C}_{60}-\mathrm{Au}$ electronic structure does not change when the solvent layer is included (Fig. 5e).

Probing atomic landscape of $2 \mathrm{D} \mathrm{MoS}_{2}$. In addition to studying the lattice structure of monoatomic graphene with a molecular STM probe in liquids, we demonstrate that this robust probe can also be extended to provide a quantitative analysis at a singleatom level of the structural composition of other 2D layered materials such as TMDs. For this, we use a $\mathrm{MoS}_{2}$ sample grown by chemical vapour deposition (CVD) on $\mathrm{Au}(111)$ (for further details on growth protocols, see 'Methods' section). The 2D-MoS is an electronic material gaining immense interest as a transistor channel material ${ }^{49}$, in biomolecular sequencing ${ }^{50}$, information storage $^{51}$ and in analytical chemistry ${ }^{52}$ for its built-in semiconducting nature and atomic structure. The as-grown $\mathrm{MoS}_{2}-\mathrm{Au}(111)$ sample is then transferred to the liquid-cell holder filled with silicone liquid and imaged using a single $\mathrm{C}_{60^{-}}$ $\mathrm{Au}$ STM probe engineered using protocols identical to those used to image graphene systems. Figure $6 \mathrm{a}$ is a large-area STM image showing regions of $\mathrm{MoS}_{2}$ layers grown on $\mathrm{Au}(111)$ terraces. Single-layer regions are identified by measuring a height of $\sim 6.8 \AA$ at the $\mathrm{MoS}_{2}$ layer and gold interface (within the region marked by the black dotted rectangle in Fig. 6a), as shown in the line section analysis (Fig. 6b). This is consistent with the expected layer thickness of TMD materials that ranges from $6.5 \AA$ to $7.1 \AA$ with a layer of metal atoms trapped between two layers of chalcogen atoms (atomic model, Fig. 6b). The measured layer thickness is also in agreement with previous low temperature STM reports on CVD grown $\mathrm{MoS}_{2}$ (ref. 53) imaged using a nonfunctionalized metal probe. Positioning the molecular STM probe in an interface region between single-layer $\mathrm{MoS}_{2}$ and the gold surface reveals the moire superstructure that stems from the lattice mismatch between the underlying $\mathrm{Au}(111)$ surface and the as-grown $\mathrm{MoS}_{2}$. A close-up view of this moiré superstructure presented in Fig. $6 \mathrm{~d}$ and the atomic structure of the moire patterns is further resolved by zooming within the same region as shown in Fig. 6e. The periodicity of the moire patterns is measured to be $32.5 \AA$ (Fig. 6e) and the presence of line-type surface defects was also observed (Fig. 6f) consistent with recent STM studies of single-layer $\mathrm{MoS}_{2}$ epitaxially grown on $\mathrm{Au}(111)$ surface $^{54}$. A spatially magnified constant-current STM image of the honeycomb type $\mathrm{MoS}_{2}$ atomic structure is shown in Fig. 6g. The position of the $S$ atoms in the hexagonal structure is better resolved by the molecular STM probe than the Mo atom sites suggesting that the electron density is dominated by toppositioned $\mathrm{S}$ atoms ${ }^{55}$. On the basis of several such highresolution images captured at low scan speeds $(1-4 \mathrm{~Hz}$ at $512 \times 512$ lines), we were able to measure a $\mathrm{MoS}_{2}$ lattice constant of $(3.2 \pm 0.1) \AA$, consistent with previous STM reports under UHV conditions using a non-functionalized STM probe. Coupled with the graphene data, this quantitative information on $\mathrm{MoS}_{2}$ indicates that a single $\mathrm{C}_{60}$-terminated STM tip can be reliably applied for atom-by-atom analytics of $2 \mathrm{D}$ nanomaterials, even when operated in liquids at room temperature. 


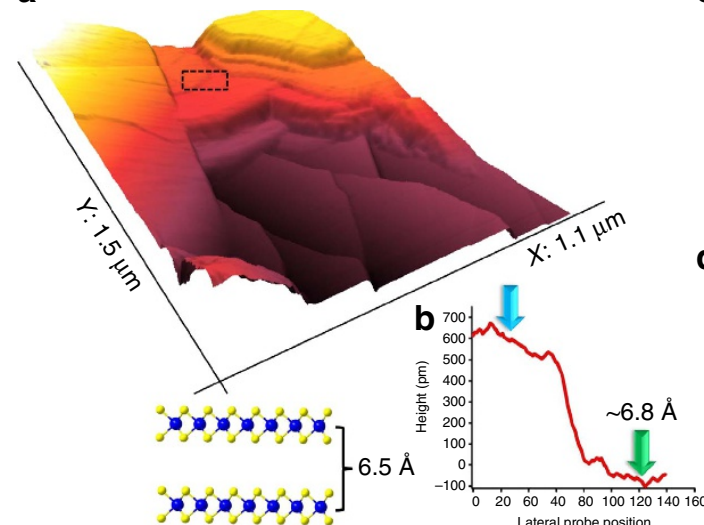

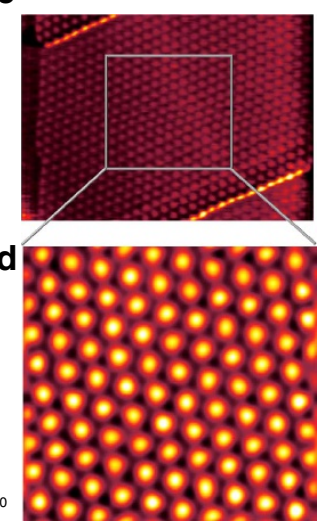

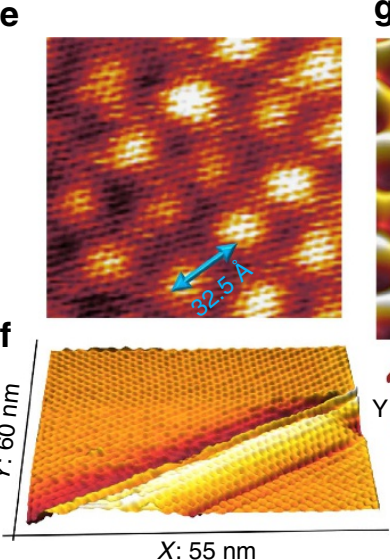

g

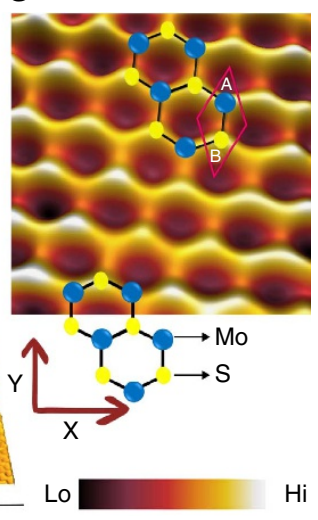

Figure 6 | Imaging the atomic lattice of 2D $\mathbf{M o S}_{\mathbf{2}}$ on $\mathbf{A u ( 1 1 1 )}$ in liquid using a molecular STM probe. (a) Large-area STM image of MoS 2 grown on $\mathrm{Au}(111)$, imaged using a $\mathrm{C}_{60}$-terminated $\mathrm{Au}$ probe in silicone liquid environment $\left(I_{t}=650 \mathrm{pA}, V_{b}=1.1 \mathrm{~V}\right)$. A line section analysis at the $\mathrm{MoS}_{2}-\mathrm{Au}$ interface shows a height difference of $\sim 6.8 \AA(\mathbf{b})$ indicating a region of single layer $\mathrm{MoS}_{2}$. The probe is then positioned on the region marked by the black rectangle in $\mathbf{a}$ and a higher-resolution constant current image is obtained (c) which shows the atomic structure of $\mathrm{MoS}_{2}$ and the bare Au surface at the edges. $\left(I_{t}=450 \mathrm{pA}, V_{b}=0.8 \mathrm{~V}\right.$, scan size: $\left.90 \mathrm{~nm} \times 70 \mathrm{~nm}\right)$. (d) Zoom-in of the region indicted by the grey square in c, revealing the moire superstructure $\left(I_{\mathrm{t}}=450 \mathrm{pA}, V_{\mathrm{b}}=-0.8 \mathrm{~V}\right.$, scan size: $\left.32 \mathrm{~nm} \times 33 \mathrm{~nm}\right)$. (e) Atomic structure of the moiré region $\left(I_{\mathrm{t}}=125 \mathrm{pA}, V_{\mathrm{b}}=-0.25 \mathrm{~V}\right.$, scan size: $\left.7.5 \mathrm{~nm} \times 7.3 \mathrm{~nm}\right)$ with a period of $32.5 \AA$. (f) STM image of a line-defect type atomic structure observed in the $\mathrm{MoS}_{2}$ domain $\left(I_{t}=180 \mathrm{pA}, V_{\mathrm{b}}=+0.5 \mathrm{~V}\right)$. $(\mathbf{g})$ Atom-resolved STM image of the hexagonal lattice structure of single-layer $\mathrm{MoS}_{2}$ with a measured lattice constant of $3.2 \pm 0.1 \AA\left(I_{t}=40 \mathrm{pA}, V_{\mathrm{b}}=+0.3 \mathrm{~V}\right.$, scan size: $2.7 \mathrm{~nm} \times 2.5 \mathrm{~nm}$ ). The scan speed parameter for $\mathbf{a}, \mathbf{c}$ and $\mathbf{e}$ was $6 \mathrm{~Hz}$ and for $\mathbf{d}, \mathbf{f}$ and $\mathbf{g}$ it was $2 \mathrm{~Hz}$ at $512 \times 512$ lines per scan.

\section{Discussion}

Taken collectively, the scanning tunnelling microscopic observations and the molecular simulations provide compelling evidence for the damping of $\mathrm{C}_{60}$ motion at the probe metal-apex in silicone oil, resulting in a stable molecular probe. Given the growing interest in analysing atomic materials under practical conditions $9,10,41,56$, our findings provide a significant step forward in atom-mapping technology in liquids at room temperature, which can be applied to examine lattice geometry, ambient compatibility and probe the impact of solvents on next generation of $2 \mathrm{D}$ semiconducting solids ${ }^{57}$. However, a deeper understanding of the influence of rotational motion of the molecule at the probe-apex will be required before these findings can be fully extended to resolve the intramolecular architecture of organic molecules with an assortment of heterogeneous chemical bonds. To address these open issues, it will require bold efforts in the future in a liquid environment, to decode the orientation of the molecule at the probe-apex ${ }^{19}$ to establish a clearer interpretation on the origin of the visualized enhancement of information content from scanning probe images.

\section{Methods}

$\mathbf{C}_{60}$ solution synthesis. $\mathrm{C}_{60}$ molecules in powder form (purchased from SigmaAldrich, 99\% purity) were solubilized (bath sonication $30 \mathrm{~s}$ ) in toluene, and a solution with $0.1 \mathrm{mM}$ concentration was prepared. One millilitre of this solution was drop-casted on a $\mathrm{Au}(111)$ surface. The excess solvent was blown dry using $\mathrm{N}_{2}$ gas and the sample was placed in a liquid cell and immediately covered with silicone oil. The silicone oil used in this study having a chemical formula of $\left.\left(\left[-\mathrm{Si}\left(\mathrm{CH}_{3}\right)_{2} \mathrm{O}\right)-\right]_{n}\right)$, where $n=22$, was purchased from Sigma-Aldrich. The CAS number is $63148-62-9$.

STM tip preparation. The tips (Au) used in this work was just mechanically cut with hand-held pliers, followed by rinsing in acetone ( $5 \mathrm{~min}$ bath sonication) and isopropyl alcohol (2 min bath sonication), blow drying with $\mathrm{N}_{2}$ gas (until dry) and followed by annealing at 50-55 degrees Celsius for $30 \mathrm{~min}$ in vacuum oven.

Fabrication and analysis of the molecular STM probe. We compared the current-voltage curves recorded using non-functionalized $\mathrm{Au}$ tips and $\mathrm{C}_{60}$-terminated STM tips (see Supplementary Fig. 1) and thus confirm (based on the evolution from metal-metal contact to a molecular signature in the IV curve) that a $\mathrm{C}_{60}$ molecule is adsorbed on the Au STM probe apex. The imaging is then temporarily halted, the molecular STM probe is withdrawn and the $\mathrm{Au}(111)$ sample is replaced by the epitaxial graphene on $\mathrm{SiC}$ sample within the liquid cell and the molecular probe is brought back into contact with the surface. An additional step to check whether the molecule is still anchored to the apex in between scans of the graphene surface is through reverse imaging in liquids. Where the molecular probe is raster scanned over a sharp defect feature acting as a secondary site on the graphene surface. This is usually a topological defect that acts as a surface tip array which then reflects the image of the molecule adsorbed on the $\mathrm{Au}$ tip apex. One such STM image is shown in Supplementary Fig 2a and Fig. 2b, where the point defects on the graphene surface aid in imaging the molecule on the tip. The underlying graphene architecture is visible and the $\mathrm{C}_{60}$ molecular shape is reflected similar to Supplementary Fig. 2a.

In addition to the lateral manipulation experiment, we have also attempted vertical manipulation, through which the molecule on surface is transferred to the tip. For this, we place the $\mathrm{Au}$ tip on top of the $\mathrm{C}_{60}$ molecule, and vary the bias voltage when keeping the tip-sample height distance constant (fixed tunnel current set point). However, for the current work, we report only the molecules transferred using the lateral manipulation approach. The success of both these experiments, lateral and vertical manipulation, is strongly dependent on the low mobility of the underlying molecule and this is achieved by trapping the molecules in a highdensity liquid such as silicone oil. To remove all other possible sources of environmental fluctuations, the measurements were conducted in next-generation noise-free laboratories, which offer vibration isolation together with precise temperature and humidity control. The specifications used include, active mechanical damping of the platform supporting the STM equipment down to $0.5 \mathrm{~Hz}$, mechanical vibration with velocity less than $500 \mathrm{~nm} \mathrm{~s}^{-1}(x, y, z)$ below $16 \mathrm{~Hz}$, electromagnetic fields with flux density less than $5 \mathrm{nT}$ RMS in an integral spectrum between 0 and $625 \mathrm{~Hz}$ and temperature stability of $0.1^{\circ} \mathrm{Ch}^{-1}$ with fluctuations of $0.5^{\circ} \mathrm{C}$ per day.

Instrument and materials details. The STM measurements were conducted in constant-current mode using Nanoscope IIIa, E-scanner, Digital Instruments. A low-current pre-amplifier circuit capable of $\sim 1 \mathrm{pA}$ sensitivity was used. The lateral STM tip drift rate is $\leq 1 \mathrm{~nm} \mathrm{~min}^{-1}$ in silicone oil medium. The STM instrument is allowed to scan with bare Au tips on lithographically designed calibration samples (Ted Pella Inc, 677-STM) for 6-8 h to reduce scanner hysteresis before mounting molecular STM probe for actual measurements on graphene. Nanoscale calibration of the scanner was performed using a freshly cleaved highly oriented pyrolytic graphite surface ( $x, y$ axes) and a $\mathrm{Au}(111)$ on mica substrate ( $z$ axis). A Teflonbased custom-built liquid-cell setup was used to support the sample substrates.

Graphene synthesis. Graphene was synthesized by epitaxial growth by thermal decomposition of hydrogen etched $4 \mathrm{H} \_\mathrm{SiC}(0001)$ surface in a furnace.

$\mathbf{M o S}_{\mathbf{2}}$ growth on Au(111). The monolayer molybdenum disulfide $\mathrm{MoS}_{2}$ samples have been obtained by CVD method on Au/mica substrates. The growth process is 
based on the gas-phase reaction between $\mathrm{MoO}_{3}$ and high-pure sulfur evaporated from solid phase. Crucible containing $\sim 5 \mathrm{mg} \mathrm{MoO}_{3}$ ( $\geq 99.998 \%$ Alfa Aesar) with $\mathrm{Au} /$ mica substrates was loaded into a three-zone furnace with a $32 \mathrm{~mm}$ outer diameter quartz tube. Located upstream from the growth substrates, a second crucible contained $\sim 350 \mathrm{mg}$ of sulfur ( $\geq 99.99 \%$ purity, Sigma-Aldrich). Ultrahigh-purity argon (Ar) was used as the carrier gas. CVD growth was performed at atmospheric pressure. The recipe of $\mathrm{MoS}_{2}$ growth is as follows: ramp the temperature to $300^{\circ} \mathrm{C}$ and set for $10 \mathrm{~min}$ at 200 s.c.c.m. of Ar flow, ramp to $700^{\circ} \mathrm{C}$ with $50^{\circ} \mathrm{C} \mathrm{min}-1$ rate and set for $10 \mathrm{~min}$ at 10 s.c.c.m. of Ar, cool down to $500^{\circ} \mathrm{C}$ and further open the furnace for rapid cooling with the increasing of $\mathrm{Ar}$ flow to 200 s.c.c.m. As a result, monolayers $\mathrm{MoS}_{2}$ flakes of the order of $1 \mu \mathrm{m}$ have been grown on $\mathrm{Au} /$ mica substrates.

Data availability. The data that support the findings of this study are available from the corresponding author on request.

\section{References}

1. Urban, K. W. Electron microscopy: the challenges of graphene. Nat. Mater. 10, 165-166 (2011).

2. Marchini, S., Günther, S. \& Wintterlin, J. Scanning tunneling microscopy of graphene on $\mathrm{Ru}(0001)$. Phys. Rev. B 76, 075429 (2007).

3. Biedermann, L. B., Bolen, M. L., Capano, M. A., Zemlyanov, D. \& Reifenberger, R. G. Insights into few-layer epitaxial graphene growth on $4 \mathrm{H}$ SiC0001 substrates from STM studies. Phys. Rev. B 79, 125411 (2009).

4. Zhao, L. et al. Visualizing individual nitrogen dopants in monolayer graphene. Science 333, 999-1003 (2011).

5. Suenaga, K. \& Koshino, M. Atom-by-atom spectroscopy at graphene edge. Nature 468, 1088-1090 (2010).

6. Novoselov, K. S. et al. Electric field effect in atomically thin carbon films. Science 306, 666-669 (2004).

7. Coleman, J. N. et al. Two-dimensional nanosheets produced by liquid exfoliation of layered materials. Science 331, 568-571 (2011).

8. Tada, T., Takemoto, S., Matsuishi, S. \& Hosono, H. High-throughput ab initio screening for two-dimensional electride materials. Inorg. Chem. 53, 10347-10358 (2014).

9. Wood, J. D. et al. Effective passivation of exfoliated black phosphorus transistors against ambient degradation. Nano Lett. 14, 6964-6970 (2014).

10. Wastl, D. S. et al. Observation of $4 \mathrm{~nm}$ pitch stripe domains formed by exposing graphene to ambient air. ACS Nano 7, 10032-10037 (2013).

11. Sonnenfeld, R. \& Hansma, P. K. Atomic-resolution microscopy in water. Science 232, 211-213 (1986)

12. Drake, B. et al. Tunneling microscope for operation in air or fluids. Rev. Sci. Instrum. 57, 441-445 (1986).

13. Itaya, K. STM studies of solid/liquid interfaces. Nanotechnology 3, 185-187 (1992).

14. Rabe, J. P. \& Buchholz, S. Direct observation of molecular structure and dynamics at the interface between a solid wall and an organic solution by scanning tunneling microscopy. Phys. Rev. Lett. 66, $2096-2099$ (1991).

15. De Feyter, S. \& De Schryver, F. C. Self-assembly at the liquid/solid interface: STM reveals. J. Phys. Chem. B 109, 4290-4302 (2005).

16. Hulsken, B. et al. Real-time single-molecule imaging of oxidation catalysis at a liquid-solid interface. Nat. Nano 2, 285-289 (2007).

17. Ciesielski, A., Palma, C.-A., Bonini, M. \& Samorì, P. Towards supramolecular engineering of functional nanomaterials: pre-programming multi-component 2D self-assembly at solid-liquid interfaces. Adv. Mater. 22, 3506-3520 (2010).

18. Ciesielski, A. et al. Dynamic covalent chemistry of bisimines at the solid/liquid interface monitored by scanning tunnelling microscopy. Nat. Chem. 6, 1017-1023 (2014).

19. Chiutu, C. et al. Precise orientation of a single C60 molecule on the tip of a scanning probe microscope. Phys. Rev. Lett. 108, 268302 (2012).

20. Gross, L. et al. Organic structure determination using atomic-resolution scanning probe microscopy. Nat. Chem. 2, 821-825 (2010).

21. Mohn, F., Gross, L., Moll, N. \& Meyer, G. Imaging the charge distribution within a single molecule. Nat. Nano 7, 227-231 (2012).

22. Schull, G., Frederiksen, T., Brandbyge, M. \& Berndt, R. Passing current through touching molecules. Phys. Rev. Lett. 103, 206803 (2009).

23. Moll, N. et al. Image distortions of partly fluorinated hydrocarbons in atomic force microscopy with carbon monoxide terminated tips. Nano Lett. 14, 6127-6131 (2014).

24. Schull, G., Frederiksen, T., Arnau, A., Sanchez-Portal, D. \& Berndt, R. Atomicscale engineering of electrodes for single-molecule contacts. Nat. Nano 6, 23-27 (2011).

25. Jiang, Y., Huan, Q., Fabris, L., Bazan, G. C. \& Ho, W. Submolecular control, spectroscopy and imaging of bond-selective chemistry in single functionalized molecules. Nat. Chem. 5, 36-41 (2013).

26. Gross, L. Recent advances in submolecular resolution with scanning probe microscopy. Nat. Chem. 3, 273-278 (2011).

27. Hapala, P. et al. Mechanism of high-resolution STM/AFM imaging with functionalized tips. Phys. Rev. B 90, 085421 (2014).
28. Sang, H. et al. Identifying tips for intramolecular NC-AFM imaging via in situ fingerprinting. Sci. Rep. 4, 6678 (2014).

29. Chiang, C.-1., Xu, C., Han, Z. \& Ho, W. Real-space imaging of molecular structure and chemical bonding by single-molecule inelastic tunneling probe. Science 344, 885-888 (2014).

30. Nadine, H. et al. Force and conductance during contact formation to a C 60 molecule. New J. Phys. 14, 073032 (2012).

31. Dimitrakopoulos, C. et al. Effect of SiC wafer miscut angle on the morphology and Hall mobility of epitaxially grown graphene. Appl. Phys. Lett. 98, 222105 (2011).

32. Kelly, K. F., Sarkar, D., Hale, G. D., Oldenburg, S. J. \& Halas, N. J. Threefold electron scattering on graphite observed with C60-adsorbed STM tips. Science 273, 1371-1373 (1996)

33. Kelly, K. F. et al. Direct observation of fullerene-adsorbed tips by scanning tunneling microscopy. J. Vac. Sci. Technol. B 14, 593-596 (1996).

34. Hugelmann, M. \& Schindler, W. Tunnel barrier height oscillations at the solid/ liquid interface. Surf. Sci. 541, L643-L648 (2003).

35. Giambattista, B. et al. Atomic resolution images of solid-liquid interfaces. Proc. Natl Acad. Sci. USA 84, 4671-4674 (1987).

36. Nirmalraj, P. et al. Nanoelectrical analysis of single molecules and atomic-scale materials at the solid/liquid interface. Nat. Mater. 13, 947-953 (2014).

37. Sass, J. K., Gimzewski, J. K., Haiss, W., Besocke, K. H. \& Lackey, D. Theoretical aspects and experimental results of STM studies in polar liquids. J. Phys. Condens. Matter 3, S121 (1991).

38. Nirmalraj, P. et al. Fingerprinting electronic molecular complexes in liquid. Sci. Rep. 6, 19009 (2016).

39. Kelly, K. F., Mickelson, E. T., Hauge, R. H., Margrave, J. L. \& Halas, N. J. Nanoscale imaging of chemical interactions: fluorine on graphite. Proc. Natl Acad. Sci. USA 97, 10318-10321 (2000).

40. Rutter, G. M. et al. Microscopic polarization in bilayer graphene. Nat. Phys. 7, 649-655 (2011).

41. Wastl, D. S., Weymouth, A. J. \& Giessibl, F. J. Atomically resolved graphitic surfaces in air by atomic force microscopy. ACS Nano 8, 5233-5239 (2014).

42. Dahal, A. \& Batzill, M. Growth from behind: intercalation-growth of twodimensional $\mathrm{FeO}$ moiré structure underneath of metal-supported graphene. Sci. Rep. 5, 11378 (2015).

43. Lui, C. H., Liu, L., Mak, K. F., Flynn, G. W. \& Heinz, T. F. Ultraflat graphene Nature 462, 339-341 (2009).

44. Xu, K., Cao, P. \& Heath, J. R. Scanning tunneling microscopy characterization of the electrical properties of wrinkles in exfoliated graphene monolayers. Nano Lett. 9, 4446-4451 (2009).

45. Fasolino, A., Los, J. H. \& Katsnelson, M. I. Intrinsic ripples in graphene. Nat. Mater. 6, 858-861 (2007).

46. Warner, J. H. et al. Bond length and charge density variations within extended arm chair defects in graphene. ACS Nano 7, 9860-9866 (2013).

47. Hapala, P., Temirov, R., Tautz, F. S. \& Jelínek, P. Origin of high-resolution IETS-STM images of organic molecules with functionalized tips. Phys. Rev. Lett. 113, 226101 (2014)

48. Cucinotta, C. S., Rungger, I. \& Sanvito, S. First principles study of electron tunneling through ice. J. Phys. Chem. C 116, 22129-22138 (2012).

49. Radisavljevic, B., Radenovic, A., Brivio, J., Giacometti, V. \& Kis, A. Single-layer MoS2 transistors. Nat. Nano 6, 147-150 (2011).

50. Feng, J. et al. Identification of single nucleotides in MoS2 nanopores. Nat. Nano 10, 1070-1076 (2015).

51. Bertolazzi, S., Krasnozhon, D. \& Kis, A. Nonvolatile memory cells based on MoS2/graphene heterostructures. ACS Nano 7, 3246-3252 (2013).

52. Pumera, M. \& Loo, A. H. Layered transition-metal dichalcogenides (MoS2 and WS2) for sensing and biosensing. Trends Anal. Chem. 61, 49-53 (2014).

53. Huang, Y. L. et al. Bandgap tunability at single-layer molybdenum disulphide grain boundaries. Nat. Commun. 6, 6298 (2015).

54. Grønborg, S. S. et al. Synthesis of epitaxial single-layer MoS2 on $\mathrm{Au}(111)$. Langmuir 31, 9700-9706 (2015).

55. Santosh, K. C., Roberto, C. L., Rafik, A., Robert, M. W. \& Kyeongjae, C. Impact of intrinsic atomic defects on the electronic structure of $\mathrm{MoS}_{2}$ monolayers. Nanotechnology 25, 375703 (2014).

56. Wastl, D. S., Judmann, M., Weymouth, A. J. \& Giessibl, F. J. Atomic resolution of calcium and oxygen sublattices of calcite in ambient conditions by atomic force microscopy using qPlus sensors with sapphire tips. ACS Nano 9, 3858-3865 (2015).

57. van der Zande, A. M. et al. Grains and grain boundaries in highly crystalline monolayer molybdenum disulphide. Nat. Mater. 12, 554-561 (2013).

\section{Acknowledgements}

We thank Leo Gross (IBM Research-Zurich) for fruitful discussions on engineering and testing the $\mathrm{C}_{60}-\mathrm{Au}$ STM probe. D.T. thanks Science Foundation Ireland (SFI) for financial support under Grant Number 11/SIRG/B2111 and computing time at the SFI/Higher Education Authority Irish Center for High-End Computing (ICHEC). 


\section{Author contributions}

P.N. conceived and performed the molecular probe-based STM measurements. D.T. designed and performed the simulation studies. C.D. synthesized the epitaxial graphene samples. D.D. and A.K. provided the $\mathrm{MoS}_{2}$ on $\mathrm{Au}(111)$ samples. All the authors contributed to the manuscript preparation.

\section{Additional information}

Supplementary Information accompanies this paper at http://www.nature.com/ naturecommunications

Competing financial interests: The authors declare no competing financial interests.

Reprints and permission information is available online at http://npg.nature.com/ reprintsandpermissions/
How to cite this article: Nirmalraj, P. et al. A robust molecular probe for Ångstrom-scale analytics in liquids Nat. Commun. 7:12403 doi: 10.1038/ncomms12403 (2016).

\section{(c) (1)}

This work is licensed under a Creative Commons Attribution 4.0 International License. The images or other third party material in this article are included in the article's Creative Commons license, unless indicated otherwise in the credit line; if the material is not included under the Creative Commons license, users will need to obtain permission from the license holder to reproduce the material. To view a copy of this license, visit http://creativecommons.org/licenses/by/4.0/

(C) The Author(s) 2016 